\title{
Solución de controversias en los sistemas de integración de Asia y África*
}

\section{Controversy resolution in Asia and Africa integration systems}

\author{
Martin Canepa** \\ * Este artículo fue desarrollado como parte del proyecto de investigación Decyt 1224 (UBA): "Sistema de Solución de Controversias \\ en el Mercosur: ¿camino a un Tribunal de Justicia?” \\ ** Maestrando en Relaciones Internacionales (UBA) - Facultad de Derecho; Abogado (UBA) - Facultad de Derecho; Jefe de \\ Trabajos Prácticos en Derecho de la Integración (UBA) - Facultad de Derecho, martincanepa@derecho.uba.ar; Buenos Aires, \\ Argentina.
}

Cómo citar: Canepa, M. (2016). Solución de controversias en los sistemas de integración de Asia y África. Inciso, 18 (2): 47-56.

Recibido: 22/01/2016| Revisado: 11/03/2016| Aceptado: 10/12/16

\section{Resumen}

Las relaciones internacionales entre los Estados implican la cooperación y el trabajo conjunto para poder lograr objetivos comunes y beneficiosos a la Comunidad Internacional en su conjunto. Supone el ejercicio de acciones de cooperación en diversos ámbitos, como el económico, social, cultural y político. La creación de estos sistemas es un fenómeno de larga data pero que en los últimos años ha tenido un crecimiento importante en ciertas regiones como por ejemplo América Latina. Otros continentes no han sido ajenos a esta corriente y también han desarrollado organizaciones que tienen por objetivo la integración de sus pueblos. Este es el caso de Asia y África, cuyo estudio acerca de estos procesos merece un lugar destacado.

Estos sistemas de integración necesitan de un mecanismo de solución de controversias para los distintos conflictos que puedan surgir. Estos métodos determinarán en gran parte ante qué tipo de sistema de integración estamos, si uno de tipo supranacional o intergubernamental. Asimismo, cada organización, atendiendo a sus particularidades y necesidades específicas va a crear las instancias que sean acordes con las diferencias que surjan de la puesta en funcionamiento de los mencionados sistemas de integración.

Palabras clave: Asia - África - Integración - Solución de Controversias

\begin{abstract}
International relations among States involve cooperation and joint work, in order to achieve common objective which benefit the International Community as a whole. It supposes cooperation actions in various scopes, such as economic, social, cultural and political. Creation of these systems is an old phenomenon, but which in the last years has grown in certain regions, such as Latin America. Other continents have not ignored this trend, and have also created organizations which purpose is integration of their people. This is the case of Asia and Africa, which study on integration deserves an outstanding place.

These integration systems need a mechanism for resolution of controversies for the various conflict which may surge. These methods will determine at a great extent, before what type of integration system we are, whether a supranational or an inter-government one. Likewise, each organization, attending to its specific particularities and needs, will create the instances according to differences surged from placement into operation the above mentioned integration systems.
\end{abstract}

Key words: Asia - Africa - Integration - Dispute Resolution 


\section{Introducción}

La integración regional se ha extendido en distintos continentes como una nueva forma de establecer relaciones entre los Estados. Los alcances de la misma pueden ir variando de acuerdo a los diversos sistemas y en particular dependiendo del desarrollo que hayan alcanzado en el tiempo. La integración puede haber desarrollado instituciones y órganos que le den supranacionalidad o basarse en un sistema de tipo intergubernamental. La diferencia entre uno y otro explica la profundidad del desarrollo del sistema y el compromiso por parte de los gobiernos en cuanto al nivel de integración que pretenden dar a las organizaciones de las cuales son parte.

El presente trabajo tiene como objetivo analizar la solución de controversias en los principales sistemas de integración de Asia y África. La integración regional ha sido poco estudiada en estos dos continentes y en general se concentra en la Unión Europea, ya que ha alcanzado el grado más desarrollado y es el único que plantea un sistema de tipo supranacional con un gran avance normativo. A su vez, la integración latinoamericana también ha sido muy prolífera y a dado lugar a la creación de un gran número de organizaciones y sistemas de integración. En primer lugar haremos una breve introducción a los métodos de solución de controversias para luego concentrarnos en el Asean, a continuación estudiaremos los dos sistemas más representativos del continente africano; la Comunidad de África Oriental y la Unión Aduanera Sudafricana y por último el sistema de integración del Consejo de Cooperación del Golfo Pérsico.

\section{Métodos de solución de controversias}

Al introducirnos en la solución de controversias entre los Estados es necesario definir cuándo nos encontramos ante una controversial internacional. La Corte Permanente de Justicia Internacional en el caso "Mavrommatis" da una definición de lo que entiende por controversial internacional: "Una controversia es un desacuerdo sobre un punto de derecho o de hecho, una posición de tesis jurídicas o de intereses entre Estados"

Los Estados disponen de distintos métodos para solucionar sus controversias. Una primera clasificación podría distinguir entre métodos no adjudicativos y métodos adjudicativos.

\section{a. No adjudicativos}

Estos métodos hacen referencia a mecanismos que no implican la adopción de una solución obligatoria para las partes, sino más bien de una recomendación, como por ejemplo, los buenos oficios, la mediación, la conciliación o la creación de una comisión investigadora. Emiten recomendaciones para las partes, las cuales son libres de adoptarlas o no. Ciertos tribunales como por ejemplo la Corte Internacional de Justicia, entre otros, pueden emitir actos no vinculantes como por ejemplo las opiniones consultivas. Lo mismo sucede con los resultados de investigaciones judiciales llevadas a cabo por la Comisión Interamericana de Derechos Humanos o del Panel de Inspección del Banco Mundial.

Un ejemplo de cómo en algunos casos resulta más sencillo resolver una controversia por medio de métodos no adjudicativos es el caso del "Canal de Beagle" entre Argentina y Chile. Para solucionar la disputa se constituyó un tribunal arbitral en febrero de 1977. El laudo dio a Chile las tres islas en disputa y trazó el límite marítimo por una línea media a través del Canal de Beagle. El resultado fue rechazado por Argentina y considerado nulo en la Declaración de Nulidad de enero de 1978. El Papa Juan Pablo II se ofreció como mediador para resolver la disputa. La oferta fue aceptada por las partes y el Papa propuso una solución en diciembre de 1980. Finalmente, con la llegada de Ricardo Alfonsín al poder, ambos países firmaron un Tratado de Paz y Amistad el 29 de noviembre de 1984, resolviendo la disputa. Fue por medio de la mediación Papal en 1994 que se logró solucionar la controversia, a pesar de haber existido laudos arbitrales con anterioridad.

\section{b. Adjudicativos}

Con el Tratado de Jay de 1794 comienza el arreglo de diferencias por medios adjudicativos. Sin embargo, se trataba de procedimientos ad hoc. Con la creación de la Corte Permanente de Justicia Internacional se inicia la institucionalización de los tribunales internacionales de carácter permanente. En el siglo $\mathrm{XX}$ vemos un crecimiento importante de los métodos 
adjudicativos tanto arbitrales como judiciales competentes en diversas materias. Estos métodos han resultado ser los medios más viables para resolver disputas internacionales.

Los métodos adjudicativos implican la adopción de una solución que resulta obligatoria para las partes en conflicto. Aquí encontramos la figura del arbitraje y de los distintos tribunales y cortes judiciales. El arbitraje y la solución judicial parecerían ser los métodos que más beneficios presentan por la obligatoriedad de los laudos y sentencias para las partes. Empero, en el ámbito del derecho internacional público general podemos ver casos en los cuales otros métodos de solución de controversias dieron mejores resultados y terminaron por zanjar definitivamente una cuestión. El fundamento es que ante la falta de un órgano o procedimiento para ejecutar los laudos o sentencias en el derecho internacional, en definitiva son los Estados los que deben cumplirlos y si no lo hacen no hay ningún órgano que los obligue en consecuencia.

\section{b.1 Arreglo Judicial}

Los tribunales judiciales toman decisiones obligatorias, su composición está dada por miembros permanentes e independientes, individuos que actúan a título personal y no representan a ningún Estado. Éstos son llamados jueces y deben cumplir con ciertos requisitos mínimos de integridad moral o antecedentes académicos, como tener las calidades necesarias para integrar las más altas esferas judiciales de sus respectivos Estados o ser expertos de reconocida competencia en el ámbito del derecho que corresponda. El resultado de la sentencia crea una nueva obligación legal para las partes, obligación de cumplir con lo dispuesto por la decisión judicial.

\section{b.2. Arbitraje}

Los miembros de estos tribunales generalmente son designados por los gobiernos de los Estados involucrados. Las partes tienen bastante control en cuanto a la composición y procedimiento ante el tribunal, aunque este control no es total. Los tribunales arbitralesse caracterizan por el hecho de que no son permanentes sino ad hoc, es decir que se crean para cado caso en particular que surgiere en relación a las controversias que se susciten.

\section{Solución de controversias en los procesos de integración}

En los sistemas de integración regional, los métodos de solución de controversias difieren dependiendo del sistema que estudiemos. Por lo general, si analizamos la historia y evolución de estos sistemas, el inicio va a estar dado por la utilización de métodos no adjudicativos con o sin elementos de tipo arbitral o jurisdiccional y en los sistemas más evolucionados ya nos encontramos con sistemas de tipojurisdiccional. La solución de controversias en el derecho internacional regional comunitario o de la integración tiene otras finalidades que van más allá de la simple solución de una disputa entre Estados como puede suceder en el ámbito del derecho internacional general. El derecho de la integración, tiene por finalidad crear un sistema común de normas aplicables a los Estados parte, consolidar las instituciones del sistema y lograr una uniformidad en las normas comunitarias. Esta tarea no puede hacerse solamente por medio de los órganos encargados de dictar las normas del sistema y de aquellos que diseñan la política común, sino que se necesita un órgano jurisdiccional que a través de la solución de conflictos determinados, en relación a la aplicación de la normativa derivada, vaya delineando el derecho común. Este tribunal, que presentará las características de permanencia y prestigio por medio de la jurisprudencia que elabore, se encargará de despejar las dudas, lagunas y conceptos de aplicación compleja o dudosa en relación al derecho comunitario.

El mejor ejemplo de cómo un tribunal de justicia de tipo supranacional fue moldeando el derecho originario y derivado de un sistema de integración, es el caso del actual Tribunal de Justicia de la Unión Europea. Su jurisdicción es obligatoria y el acceso está garantizado a los Estados, Instituciones de la Unión y los particulares. Su función básica es la de hacer cumplir el derecho de la Unión, interpretarlo y aplicarlo. Así lo establece el Tratado de la Unión Europea en su artículo 19: "El Tribunal de justicia de la Unión Europea comprenderá el Tribunal de justicia, el Tribunal General y los Tribunales especializados. Garantizará el respeto del Derecho en la interpretación y aplicación de los Tratados".

Dentro de la competencia del Tribunal existe un elemento de suma importancia, llamado cuestión 
prejudicial que ha servido para dar uniformidad al derecho de la Unión en el territorio de los Estados parte. El Tribunal es competente para pronunciarse con carácter prejudicial:

a) Sobre la interpretación de la Constitución

b) Sobre la validez e interpretación de los actos adoptados por las instituciones, órganos $\mathrm{u}$ organismos de la Unión.

No se trata de un procedimiento autónomo, sino que es un incidente prejudicial que tiene origen en un proceso contencioso dentro del territorio de un Estado parte. Sirve como un mecanismo de control indirecto de la legalidad de la Unión y como instrumento de colaboración entre los Tribunales nacionales y el Tribunal de justicia de la Unión Europea. El artículo 267 del Tratado de Funcionamiento de la Unión Europea establece que: "Cuando se plantee una cuestión de esta naturaleza ante un órgano jurisdiccional de uno de los Estados miembros, dicho órgano podrá pedir al Tribunal que se pronuncie sobre la misma, si estima necesaria una decisión al respecto para poder emitir su fallo". Es decir, que si se presenta alguno de los casos mencionados en los puntos a) o b) el Tribunal interno tiene la posibilidad de acudir al Tribunal de Justicia de la Unión para dar más claridad a la cuestión planteada. La obligatoriedad de la sentencia del Tribunal de justicia de la Unión en relación a la interpretación o validez o de una norma de derecho comunitario, ya sea originaria o derivada, brinda más certeza y uniformidad al derecho supranacional.

\section{Análisis en particular}

\section{Asean}

La asociación de Naciones del Sudeste Asiático fue creada en el año 1967. Esta organización tiene como finalidad lograr una mayor integración entre los Estados miembros para poder de esta manera llegar a establecer una zona de libre comercio, reducir las tarifas aduaneras, acelerar el crecimiento económico de la región y mantener la paz y la estabilidad regionales. Son parte de este sistema de integración regional Indonesia, Malasia, Filipinas, Singapur, Tailandia, Brunei, Vietnam, Laos, Birmania y Camboya.
En relación a la solución de controversias que pudieren surgir del mismo proceso, el articulo 22 (1) de la Carta fundacional ${ }^{1}$, dispone que como regla general los Estados miembros procurarán resolver las controversias de manera pacífica, en un tiempo prudencial a través del diálogo, consultas y negociación. A su vez, los Estados en disputa pueden acordar acudir a los buenos oficios, conciliación o mediación en cualquier momento de la controversia. Consecuentemente, pueden solicitar al Director del Asean o al secretario general que brinde los buenos oficios o participe en los procedimientos de conciliación o mediación. En este sentido, siguiendo al Profesor Walter Woon ${ }^{2}$ existió una discusión en el seno de las "Tareas de alto nivel", acerca de si la Carta debería dar competencia al Director o al secretario general para ofrecer sus buenos oficios, conciliación o mediación sin ser requeridos por las partes. Finalmente, se decidió que sería mejor que las partes pidieran su ayuda en el caso que surgiera alguna disputa.

El artículo 24 dispone que aquellas disputas relativas a instrumentos específicos del Asean serán resueltas a través de los mecanismos y procedimientos establecidos en dichos instrumentos. Posteriormente en el inciso 2 del mismo artículo se especifica que las disputas que no estén relacionadas con la interpretación o aplicación de ningún instrumento del Asean serán resueltas en forma pacífica de acuerdo al Tratado de Amistad y Cooperación del Sudeste Asiático $(\mathrm{TAC})^{3}$ y a sus reglas de procedimiento. Este instrumento, dispone que aquellas disputas que pongan en peligro la paz, serán remitidas al Alto Consejo conformado por representantes de cada Estado miembro, siempre que los Estados acuerden someter la controversia al Consejo.

A su vez el inciso 3 determina que salvo disposición en contrario, las disputas relativas a la interpretación o aplicación de los acuerdos económicos del Asean deberán encontrar una solución en las reglas que crea el Protocolo sobre mecanismo reforzado de solución de controversias (Protocolo de Vientiane) ${ }^{4}$.

1. Asean Charter, 20 de Noviembre, 2007, Singapur.

2. Profesor Walter Woon, attorney-general, Asean Law Association, $10^{\text {th }}$ Assembly, Singapore.

3. Treaty of Amity and Cooperation in Southeast Asia (TAC), 24 de Febrero de1976, Bali, Indonesia.

4. Protocolo sobre mecanismo reforzado de solución de controversias, Vien- 
El Protocolo de Vientiane, es el más importante de todos los mecanismos de solución de controversias diseñado en el ámbito del Asean. Básicamente cubre las disputas relativas a acuerdos de tipo económico. En términos generales, el Protocolo establece la constitución de paneles que estudian la controversia y expresan sus conclusiones al finalizar el proceso. Las mismas son remitidas a la Reunión Superior Económica Oficial que deberá tomar una determinación en última instancia. El panel debe considerar la naturaleza de la disputa entre las partes y decidir cómo resolverla.

El artículo 25 establece que en los casos en los que no existe un mecanismo específico para la resolución de una determinada controversia en relación a la interpretación o aplicación de la Carta y otros instrumentos del Asean, deberán crearse instrumentos concretos, incluyendo el arbitraje. Esta disposición, habilita la creación de un Tribunal de tipo judicial, el cual no ha sido creado hasta el momento.

Por su parte el artículo 26 dispone que si una disputa continua sin ser resuelta luego de la aplicación de los procedimientos previstos en la Carta, la disputa deberá ser remitida a la Cumbre del Asean, para su consideración.

En relación al cumplimiento, el secretario general asistido por la Secretaría u otro órgano designado del Asean, deberá controlar el cumplimento de las recomendaciones o decisiones que resulten del mecanismo de solución de controversias y enviar un reporte a la Cumbre con sus respectivas consideraciones. De la misma manera, cualquier Estado miembro que se vea afectado por el no cumplimiento de alguna recomendación o decisión que resulte del mecanismo de solución de controversias podrá remitir el asunto a la Cumbre para su decisión.

Por último, el artículo 28 establece, que salvo disposición en contrario, los Estados miembros tienen el derecho de recurrir a los modos pacíficos de solución de controversias contenidos en el artículo 33 (1) de la Carta de las Naciones Unidas o a cualquier otro instrumento internacional del cual fueran parte los Estados miembros.
Es importante destacar que la Carta en sí misma, no contiene ningún mecanismo para resolver las disputas relativas a la interpretación de la Carta. Si se presentara el caso, se aplicaría el articulo 51 (1), el cual dispone que la Secretaria deberá realizar la interpretación en cuestión.

En definitiva, el sistema creado por esta organización regional brinda un mecanismo de solución de controversias que incluye, la negociación, la conciliación, los buenos oficios y que prevé la posibilidad de establecer tribunales de tipo arbitral, reservando ciertos mecanismos para casos determinados. El sistema diseñado revela el carácter intergubernamental de la organización, aunque vislumbra la creación de un futuro tribunal de justicia.

\section{La Comunidad de África Oriental}

La Comunidad de África Oriental (EAC - East African Community) es una organización regional de tipo intergubernamental conformada por la Republica de Burundi, Kenia, Ruanda, Tanzania y Uganda. La sede se encuentra en Arusha, capital de Tanzania. La Comunidad volvió a funcionar el 30 de noviembre de 1999, cuando el Tratado 5 para su restablecimiento fue firmado. Entró en funcionamiento el 7 de julio de 2000. El proceso de integración comenzó en 1993, con el objetivo de lograr un programa de cooperación política, económica, social y cultural, de defensa, tecnología, investigación, seguridad, legal y judicial.

La Corte de justicia (La Corte) es uno de los órganos establecidos de acuerdo al artículo 9 del Tratado para el establecimiento de la Comunidad de África Oriental. Es importante mencionar la desaparecida Corte de Apelaciones, antecesora de la actual Corte de Justicia, la cual funcionaba como una corte de apelaciones respecto de las decisiones de la Cortes nacionales tanto en materia civil como penal excepto en cuestiones de derecho constitucional y el delito de traición a la patria en relación a Tanzania. La actual Corte de justicia tiene como principal función asegurar el cumplimiento del derecho en la interpretación y aplicación del Tratado de creación.

5. Tratado de creación de la Comunidad de África Oriental, Arusha, Tanzania, 30 de noviembre de 1999.

tiane, Laos, 2004 
En el año 2001 comenzó a funcionar. El trabajo de la Corte durante el periodo de transición es de tipo ad hoc hasta que el Consejo de Ministros determine que existe suficiente trabajo como para que funcione en forma permanente. En consecuencia, los jueces no están obligados durante este período, a residir en forma permanente en la ciudad de Arusha, donde se encuentra la sede temporaria de la Corte, sin embargo, deben asistir a la sede cuando existiere un caso judicial.

\section{a. Sede}

La ciudad de Arusha es la sede temporaria de la Corte hasta que la Cumbre de ministros determine la sede permanente. Las Cortes nacionales de los Estados miembros servirán como registros de las actividades de la Corte.

\section{b. Composición}

Los jueces de la Corte son designados por la Cumbre de ministros de entre los jueces nacionales de los Estados parte o juristas de reconocida competencia y el secretario es designado por el Consejo de ministros. El presidente y vicepresidente también son nombrados por la Cumbre de ministros de entre los jueces de la Corte. Originalmente estará compuesta por seis jueces, dos de ellos serán originarios de los tres estados fundadores de la Comunidad, es decir, Kenia, Tanzania y Uganda. Actualmente la Corte está compuesta por diez jueces. De acuerdo a la reforma del Tratado de establecimiento de la Comunidad de África Oriental que fue llevada a cabo el 14 de diciembre de 2006 y el 20 de agosto de 2007, la Corte fue reformada y dividida en dos secciones: una primera instancia y una instancia de apelación. A su vez la Corte se encuentra dividida en tres comités:

- Finanzas y administración

- Formación y Relaciones públicas

- Reglamento de la Corte

\section{c. Jurisdicción}

La Corte tiene competencia para los siguientes casos:

- Disputas en la interpretación y aplicación del Tratado.
- Disputas entre la Comunidad y sus empleados surgidas de términos y condiciones de empleo o en relación a la interpretación y aplicación del derecho y regulación del personal de la Comunidad.

- Disputas entre los Estados parte en relación al Tratado, siempre que la disputa sea sometida a la Corte a través de un acuerdo especial.

- Disputas surgidas de una cláusula arbitral contenida en un contrato o acuerdo que confiere jurisdicción a la Corte del cual es parte la Comunidad o cualquiera de sus instituciones.

- Disputas surgidas de una cláusula arbitral contenida en un contrato o acuerdo comercial por medio del cual las partes dan competencia a la Corte para resolver la controversia.

- La jurisdicción de la Corte puede ser extendida a los derechos humanos, cuando oportunamente lo determine el Consejo de Ministros.

d. Acceso

Tienen acceso a la jurisdicción de la Corte:

- Los Estados parte: un Estado parte puede hacer una denuncia ante la Corte si considera que otro Estado Parte o un órgano o institución de la Comunidad no ha cumplido una obligación o ha violado alguna disposición del Tratado. A su vez, un Estado parte también podrá solicitar a la Corte que determine la legalidad de un acto, reglamento, directiva, decisión o acto que haya sido sancionado por medio de abuso de poder, que sea ilegal o violatorio de las disposiciones del Tratado.

- El secretario general: si un Estado parte no cumple con alguna de sus obligaciones establecidas por el Tratado, el secretario general puede remitir el asunto a la Corte.

- Personas físicas y jurídicas: una persona física o jurídica, residente en cualquiera de los Estados parte, puede controvertir la legalidad de cualquier acto, reglamento, directiva o decisión de un Estado parte o institución de la Comunidad que viole alguna disposición del Tratado. 


\section{e. Opiniones consultivas}

La Cumbre de Ministros, el Consejo o un Estado parte pueden solicitar a la Corte que emita una opinión consultiva relativa a una cuestión de derecho que surja del Tratado y que afecte a la Comunidad.

El tribunal de justicia creado para resolver controversias en el ámbito de este sistema de integración presenta características propias de un sistema supranacional, aunque por el momento si bien ya comenzó a funcionar, su actividad es de tipo ad hoc, resolviendo casos para cada situación que se presente, lo que implica que su funcionamiento no es permanente. Sin embargo, la iniciativa de los Estados partes en el sentido de la creación de una Corte de justicia es destacable ya que implica la voluntad de salir del tipo intergubernamental de integración e involucrarse en un proceso más profundo y desarrollado.

\section{Unión Aduanera Sudafricana (SACU - Southern African Customs Union)}

El acuerdo de Unión aduanera del año $2002^{6}$ entre la República de Botsuana, el Reino de Lesoto, Namibia, Sudáfrica y el Reino de Suazilandia se propone reanudar el proceso de integración iniciado en 1969 y adecuarlo a las nuevas exigencias y características de los Estados miembros. El mismo acuerdo establece la creación de un Tribunal ad hoc para resolver las disputas que tuvieren relación con el proceso de integración, sin embargo todavía no ha entrado en funcionamiento.

El Acuerdo del año 2002, en su artículo 13 establece la creación de un Tribunal. Toda disputa relacionada con la interpretación o aplicación del Tratado o a solicitud del Consejo, será dirimida por un Tribunal ad hoc. El mismo estará compuesto por tres miembros, excepto que el Consejo determine otra forma de composición. Decidirá por mayoría de votos y su decisión será definitiva y obligatoria. A solicitud del Consejo, dictará recomendaciones. En cualquier caso sometido al Tribunal, las partes elegirán a los miembros del Tribunal de una lista de nombres aprobaba previamente por el Consejo y registrada ante la Secretaría. Los Estados miembros

6. Southern African Customs Union Agreement, Gaborone, Botsuana, 2012. se comprometen a intentar resolver las disputas por medio de medios amistosos antes de someterla al Tribunal. La Secretaria asistirá al Tribunal en su trabajo y el Tribunal dictará sus propias reglas de procedimiento.

Este acuerdo no se encuentra aún en un gran desarrollo, ya que solo prevé la constitución de un tribunal arbitral y se trata de un sistema de solución de controversias que por el momento no vislumbra el establecimiento de una Corte de justicia, lo que lo asemeja a otros tipos de sistemas de integración de tipo intergubernamental como el Consejo de Cooperación del Golfo Pérsico o el Mercosur.

\section{Consejo de cooperación del Golfo Pérsico}

El Consejo $^{7}$ se encuentra conformado por los Emiratos Árabes Unidos, Bahréin, el Reino de Arabia Saudita, Omán, Qatar y Kuwait. Sus objetivos son la coordinación e integración en el ámbito económico y financiero, comercial, aduanero, en las comunicaciones, educación y cultural.

El órgano de mayor jerarquía dentro de la organización es el Consejo Supremo, que se encuentra formado por los seis Estados que participaron de la Reunión de Ministros de Relaciones Exteriores desarrollada en Riad el 4 de febrero de 1981. Una de las funciones de este Consejo consiste en aprobar las reglas de procedimiento de la Comisión para el arreglo de diferencias y nombrar a los miembros de la misma. El artículo 10 de la Carta de constitución de la Organización, regula el funcionamiento de la Comisión para el arreglo de diferencias y establece:

- El Consejo de Cooperación tendrá una comisión que se denominará "Comisión para el arreglo de diferencias", la cual será coordinada por el Consejo Supremo.

- El Consejo Supremo establecerá la composición de la Comisión para cada caso que se presente, de manera ad hoc, de acuerdo a la naturaleza de la disputa.

- Si surgiere una disputa sobre la interpretación o aplicación de la Carta y la disputa no es

7. Carta del Consejo de Cooperación del Golfo Pérsico, Abu Dhabi, Emiratos Árabes Unidos, 25 de Mayo de 1981. 
resuelta por el Consejo Ministerial o el Consejo Supremo, entonces este último podrá solicitar que la disputa sea resuelta por Comisión para el arreglo de diferencias.

- La Comisión transmitirá sus recomendaciones u opinión, según corresponda, al Consejo Supremo.

\section{a. Centro de Arbitraje}

Los Estados miembros del Consejo de Cooperación establecieron un Centro de Arbitraje Comercial $^{8}$ con sede en Bahréin. En virtud del artículo 2 de la mencionada Carta, el Centro es competente para examinar disputas entre nacionales de alguno de los Estados miembros o entre ellos y otras personas físicas o jurídicas respecto de conflictos de carácter comercial que surjan de la aplicación de las disposiciones del Acuerdo Unificado Comercial y las resoluciones emitidas para su implementación, siempre que ambas partes acuerden por medio de un contrato celebrado por escrito o en un acuerdo posterior, someter la controversia al Centro.

Los principales órganos del Centro son:

- La Junta Directiva

- Secretario General

- Tribunal Arbitral

- Secretaría del Tribunal Arbitral

En virtud del artículo 10 de la Carta de constitución del Centro, el Tribunal estará constituido por uno o tres árbitros de acuerdo a lo que conjuntamente hayan acordado las partes. En el caso de que no se logre un acuerdo en cuanto a la elección, serán aplicables las Reglas de Procedimiento elaborados por la Junta Directiva. El Centro deberá proporcionar una lista de árbitros preparada por las cámaras de comercio e industria de cada uno de los Estados miembros del Consejo y las partes en una controversia tendrán acceso a dicha lista para seleccionar a los árbitros siendo también posible seleccionar árbitros que no se encuentren en la mencionada lista. Los árbitros deberán ser abogados, jueces o personas que

8. Carta de creación del Centro de Arbitraje Comercial, Riad, Reino de Arabia Saudita, diciembre de 1993. posean una amplia experiencia y conocimiento en materia comercial, industrial o financiera. Deberán gozar de alta integridad moral, buena conducta e independencia.

En relación al derecho aplicable, el artículo 12 de la mencionada Carta dispone que las partes tienen libertad para decidir el derecho aplicable. En el caso de que las partes no hayan acordado el derecho aplicable en el contrato o en el acuerdo arbitral, los árbitros deberán aplicar el derecho determinado por las reglas del conflicto de leyes que consideren apropiadas, ya sea el derecho del lugar donde fue celebrado el contrato, el derecho del lugar donde el contrato fue ejecutado, el derecho del lugar donde debe ser ejecutado o cualquier otro derecho sujeto a los términos del contrato y las reglas y costumbres del derecho internacional.

Se establece que el laudo del Tribunal será definitivo y no sujeto a recurso alguno ante ningún órgano judicial. Su cumplimiento es obligatorio para las partes y podrá ejecutarse ante la autoridad judicial correspondiente de uno de los Estados parte.

\section{b. Unión Monetaria}

En virtud del Acuerdo 9 que prevé la creación de una Unión Monetaria, la cual todavía no se ha constituido, se dispone en el artículo 24 relativo a la solución de controversias, que los Estados miembros ante una disputa relativa a la interpretación o implementación de alguna de las disposiciones del acuerdo deberán buscar una solución a través de métodos amistosos. Si no se encontrare una solución, la disputa será resuelta por medio del arbitraje de acuerdo a los procedimientos que oportunamente se establezcan.

c. Medidas antidumping, compensatorias y de salvaguardia

Por su parte, las reglas adoptadas por el Consejo Supremo sobre medidas antidumping ${ }^{10}$ establecen la creación de un Comité Permanente compuesto por representantes de cada de uno de los gobiernos de los Estados parte. En virtud del artículo 9.6 de las reglas

9. Acuerdo de creación de la Unión Monetaria, Mascat, Omán, 30 de diciembre de 2008 .

10. Reglas sobre medidas antidumping, compensatorias y de salvaguardia, 24 Sesión del Consejo Supremo, Kuwait, 21-22 de diciembre de 2003. 
mencionadas con anterioridad, el Comité, en el marco de sus funciones, deberá proponer recomendaciones apropiadas para la solución de controversias que surjan entre los Estados miembros sobre la interpretación de estas reglas. Por otro lado, también se prevé la creación de un Comité de Cooperación Industrial, el cual deberá tomar una decisión final en relación a la solución de controversias sobre la interpretación de las reglas relativas a medidas antidumping, compensatorias o de salvaguardia, que pudieren surgir entre los Estados parte.

\section{d. Conservación de la vida salvaje}

La Convención ${ }^{11}$ para la conservación de la vida salvaje también prevé un mecanismo especial para la solución de controversias que surjan de la interpretación o aplicación de la misma. En consecuencia, el artículo 6 dispone la creación de un Comité Permanente conformado por representantes de los Estados parte, el cual tendrá entre otras funciones, la de facilitar la solución amistosa de cualquier disputa que surja entre las partes en el marco de la implementación de la Convención de acuerdo al artículo 9. Este último establece que el Comité deberá realizar todos los esfuerzos para que las controversias sean resueltas de manera amistosa. En el supuesto de que fuere imposible resolver la controversia en esta instancia o a través de negociaciones directas, el Comité elevará la cuestión a los Ministros correspondientes.

Luego de haber analizado los principales mecanismos de solución de controversias en el ámbito del Consejo de Cooperación del Golfo Pérsico, podemos observar que nos encontramos ante una organización de tipo intergubernamental, donde no existe un Tribunal de justicia supranacional sino que se han diseñado distintos métodos de solución de conflictos que van desde las negociaciones directas hasta la constitución de tribunales arbitrales para las controversias que surjan en los distintos ámbitos de aplicación y desarrollo del sistema de integración desarrollado en esta región.

11. Convención para la conservación de la vida salvaje y su hábitat natural, Mascat, Omán, 30 de diciembre de 2001.

\section{Conclusión}

El análisis del sistema creado por cada uno de estos procesos de integración en el ámbito asiático y africano, pone de manifiesto que ninguno de ellos puede por el momento considerarse como un sistema de integración supranacional ya que la gran mayoría no tiene como método de solución de conflictos un tribunal de justica, sino que se basan en sistemas de tipo no adjudicativo, incluyendo entre otros la mediación, la conciliación, los buenos oficios o las negociaciones. Aquellos que poseen métodos de tipo adjudicativo, han creado instancias arbitrales o de paneles y en los casos en que encontramos Tribunales de Justicia, estos últimos o bien no se encuentran operativos o requieren aun de ciertos mecanismos de desarrollo para que puedan funcionar plenamente y no se conviertan en tribunales que actúen solo de manera ad hoc. Lo que resulta interesante de destacar es que algunos sistemas como el Asean o el Consejo de Cooperación del Golfo Pérsico, han elaborado distintos sistemas de solución de controversias de acuerdo al tipo de conflicto que se presente. Cada acuerdo que regula una determinada materia tiene su propio sistema de solución de controversias. El aspecto negativo de esta forma de regulación es que podrían existir tantos sistemas de solución de conflictos como acuerdos que regulen distintas actividades. Un sistema de integración para poder desarrollarse y lograr un efectivo cumplimiento de sus normas, de manera tal que el desarrollo normativo no sea solo una tarea utópica, necesita de un Tribunal Judicial, que aplique e intérprete de manera uniforme las normas del derecho primario y del derivado de este proceso y que en consecuencia cree una base sólida de seguridad jurídica.

\section{Referencias bibliográficas}

Asean Economic Community 2015: Progress and Key Achievements, Jakarta: ASEAN Secretariat, November 2015. En: http:// Www.asean.org/storage/images/2015/ November/aec-page/AEC-2015-Progressand-Key-Achievements.pdf 
Askari Rizvi, H. (1982), "Gulf Cooperation Council”, Pakistan Horizon, Pakistan Institute of International Affairs vol.35, $\mathrm{n}^{\circ} 2$ (Second Quarter 1982), 29-38.

Barboza, J. (2001) Derecho Internacional Público, Buenos Aires: Zavalia, Segunda edición.

Chittharanjan F.A. (2003) "Jurisdiction of International Tribunals", Netherlands: Kluwer Law International.

De La Fayette, L.A. (2009) "Beagle Channel Dispute", Max Planck Institute for Comparative Public Law and International Law, Heidelberg: Heidelberg and Oxford University Press.

Denner, W. (2009) "Trade remedies and safeguards in southern and eastern Africa" en Monitoring Regional Integration in Southern Africa Yearbook, vol.9, Trade Law Centre for Southern Africa (tralac), Stellenbosch, South Africa, 43-75.

Farajallah, S.B (1991) "Le Conseil de coopération des Etats arabes du Golfe", Collected Courses of The Hague Academy of International Law, 228, 9-182.

Frimpong Oppong, R. (2011) "Legal Aspects of Economic Integration in Africa", Cambridge: University Press.

Gutierrez Posse, H. (1983) “Algunas consideraciones sobre la solución pacífica de controversias internacionales", Revista Lecciones $y$ Ensayos, 4, 33-45.

Pizzolo, C. (2010) Derecho e Integración Regional: Comunidad Andina, Mercosur, SICA, Unión Europea, Buenos Aires: EDIAR $1^{\mathrm{a}}$ edición.

Razeen, S. (2010) "Regional Economic Integration in Asia: the track record and prospects", en ECIPE, $\mathrm{N}^{\circ} 2$, Bruselas. En: http://www.ecipe. org/app/uploads/2014/12/regional-economic-integration-in-asia-the-track-record-and-prospects.pdf

Robson, P. (2011) "Economic Intergation in Africa", Routledge: Routledge Library editions.

Romano, C.P.R. (2010) "A Taxonomy of International Rule of Law Institutions", en Journal of International Dispute Settlement, $2,(1)$.

Terms of Reference of Asean Intergovernmental Commission on Human Rights, Jakarta: ASEAN Secretariat, May 2014. En: https://www 1.umn.edu/humanrts/ research/Philippines/Terms $\% 20$ of\% 20 Reference $\% 20$ for $\% 20$ the $\% 20$ ASEAN $\% 20$ Inter-Governmental\%20CHR.pdf

Vinuesa, R. (1983) "La solución pacífica de controversias entre Estados", Revista Lecciones y Ensayos, 4, 7-32.

Wilson, R. (2013) "Economic Development in the Middle East", Abingdon, Oxon: Routledge. 